\title{
World Review
}

Int J Epidemiol 2000;29:933-940

\section{Effectiveness of Incidence Thresholds for Detection and Control of Meningococcal Meningitis Epidemics in Northern Togo}

Anne-Valérie Kaninda, François Belanger, Rosamund Lewis, Essosolem Batchassi, Aristide Aplogan, Yénou Yakoua, Christophe Paquet

Background: Early outbreak detection is necessary for control of meningococcal meningitis epidemics. A weekly incidence of 15 cases per 100,000 inhabitants averaged over 2 consecutive weeks is recommended by the World Health Organization (WHO) for detection of meningitis epidemics in Africa. This and other thresholds are tested for ability to predict outbreaks and timeliness for control measures. Methods: Meningitis cases recorded for 1990-1997 in health centres of northern Togo were reviewed. Weekly and annual incidences were determined for each district. Ability of different weekly incidence thresholds to detect outbreaks was assessed according to sensitivity, specificity, and positive and negative predictive values. The number of cases potentially prevented by reactive vaccination in 1997 was calculated for each threshold. Results: Outbreaks occurred in 1995-1996 and in 1996-1997. The WHO-recommended threshold had good specificity but low sensitivity. Thresholds of 10 and 7 cases per 100,000 inhabitants in one week had sensitivity and specificity of $100 \%$ and increased the time available for intervention by more than one or two weeks, respectively. A maximum of $65 \%$ of cases could have been prevented during the 1997 epidemic, with up to $8 \%$ fewer cases prevented for each week of delay in achieving vaccine coverage. Conclusions: In northern Togo, thresholds of 7 or 10 cases per 100,000 inhabitants per week were excellent predictors of meningitis epidemics and allowed more time for a reactive vaccination stragegy than current recommendations.

Int J Epidemiol 2000;29:941-947

High Prevalence of Congenital Toxoplasmosis in Brazil Estimated in a 3-Year Prospective Neonatal Screening Study

Eurico Camargo Neto, Elaine Anele, Rosélia Rubim,

Adriana Brites, Jaqueline Schulte, Daniela Becker,

Tamara Tuuminen

Background: A pilot neonatal screening programme revealed a high (approximately 1 per 4,800 live births) prevalence of congenital toxoplasmosis (CT) in the State of Rio Grande do Sul, Brazil. The purpose of this paper was to estimate in a larger prospective study the prevalence of CT in the country. Methods: At the beginning of the study, an in-house indirect enzyme immunoassay (EIA) was used, to be later replaced with a commercial capture IgM fluorometric enzyme immunoassay (FEIA). Both methods detect specific antiToxoplasma gondii IgM-class antibodies eluted from dried blood spots. Results: Of the total of 140,914 samples received from all over the country, 47 cases were identified and confirmed as CT. This finding suggests a prevalence of 1 per 3,000 live births. Of the 47 patients, only eight (17\%) had clinical manifestations; two had intracranial calcifications, four had retinal scars, one had an intracranial calcification and retinal scars, and one had hepatosplenomegaly with lymphoadenopathy. The testing was paid for by the patients' families who volunteered for the study and gave their informed consent. Conclusion: The 3-year prospective study using sensitive detection methods, reliable confirmation, and feedback from clinicians showed that CT has an extraordinarily high prevalence in Brazil, in fact the highest ever reported in the world. Although the long-term efficacy of treatment of CT has not been well documented, in view of the availability of reliable diagnostics, confirmation and monitoring, functional logistics, and networking for screening, the insidious nature of the sequelae and the very high prevalence of the disease, neonatal screening for $\mathrm{CT}$ should be considered an alternative to no screening at all.

Arq Neuropsiquiatr 2000;58:826-839

\section{Trail Making and Cognitive Set-Shifting}

Ricardo de Oliveira-Souza, Jorge Moll, Leigh J Passman, Fernando Cimini Cunha, Flávia Paes, Marcus Vinicius Adriano, Fátima Azevedo Ignácio, Rogério Paysano Marrocos

We tested the hypothesis that Part B of the Trail Making Test (TMT) is a measure of cognitive set-shifting ability in 55 normal subjects with the conventional (written) TMT and a verbal adaptation, the 'verbal TMT' (vTMT). The finding of a significant association between Parts B of TMT and vTMT $(r=0.59, \mathrm{p}<0.001)$, after correcting for age and education, supports the view that Part B of TMT is a valid measure of the ability to alternate between cognitive categories.

\section{KARGER}

Fax + 41613061234

E-Mail karger@karger.ch

www. karger.com

\section{(C) 2001 S. Karger AG, Basel}

Accessible online at

www. karger.com/journals/ned 


\section{Neurocysticercosis: A Clinical and Pathological Study of 27 Necropsied Cases (in Portuguese)}

Mário Rodrigues Montemór Netto,

Emerson Leandro Gasparetto, Leonardo Nercolini Faoro, Jorge Sergio Reis Filho, Guilherme Sandrini de Toni,

Arnolfo de Carvalho Neto, Luiz Fernando Bleggi Torres

Neurocysticercosis is the most frequent and widespread neuroparasitosis of the human being. The development of brain and leptomeningeal lesions, with subsequent symptoms, are mainly related with the immune status of the host, and to the number and evolutional phase of the parasites. We present the pathological findings in 27 necropsies of patients with neurocysticercosis, which accounted for $31 \%$ of the necropsies. $77 \%$ of the patients were male and the age ranged from 18 to 85 years. In $26 \%$ there was previous history of alcoholism. Clinicopathological study showed that $50 \%$ o the cases were classified as asymptomatic form, $11 \%$ epileptic form, $11 \%$ intraventricular form and $11 \%$ combined form. $33 \%$ of the patients presented seizures as a factor of aggravation of the clinical picture. There was a single cysticercus in $60 \%$ of the cases, the cellulosae form present in $82 \%$ and the racemous form in $7 \%$ of the cases; the remaining $11 \%$ had both forms present. In $30 \%$ of the patients the cause of death was directly related with the presence of the cysticercus in the central nervous system. Our findings confirm the high morbidity of this disease.

Arq Neuropsiquiatr 2000;58:890-896

\section{Malformations of the Central Nervous System: Analysis of 157 Pediatric Autopsies (in Portuguese)}

Lúcia de Noronha, Fabiola Medeiros,

Vanessa dello Mônaco Martins, Gilberto Antunes Sampaio, Maria José Serapião, Gilda Kastin,

Luiz Fernando Bleggi Torres

The malformations of the central nervous system affect about 5 to 10 children per 1,000 births. We studied the central nervous system malformations in 5,837 pediatric autopsies performed in the Sector of Anatomic Pathology, Hospital de Clinicas-UFPR, between 1960 and 1995 . There were 157 central nervous system malformations (2.69\%), the commonest were neural tube defects (61\%): 47 cases of anencephaly and 45 cases classified in the group of myeloencephaloceles. The anomalies of the prosencephalic evagination corresponded to $8 \%$ of all central nervous system malformations, with seven cases of holoprosencephaly. Posterior fossa malformations occurred in 3\%, with three cases of Arnold-Chiari. In the present study, the mortality rate due to central nervous system malformations was higher in the neonatal period.

\section{Consensus Conference: Parkinson's Disease: Diagnostic and Therapeutic Criteria (in French)}

Proceedings of a conference held at the Charcot Amphithéâtre, Pitié Salpêtrière Hospital, Paris, March 3, 2000

The conference was organized by the French Federation of Neurology with participation of experts in neurology, movement disorders, neurosurgery, psychiatry, physical medicine and rehabilitation, clinical neurophysiology, pharmacology and internal medicine. The consensus conference addressed the following topics:

1 Diagnostic Criteria and Complementary Explorations Clinical diagnostic criteria in Parkinson's disease and parkinsonian syndromes

Place of paraclinical examinations in Parkinson's disease

2 Evaluation Scales for Parkinson's Disease

Different scales for evaluation of Parkinson's disease, rating scales and questionnaires

Limitations and advantages of rating scales

3 Therapeutic Strategies for Early Stage Parkinson's Disease

Dopa therapy

Dopaminergic agonists

Other treatments

4 Therapeutic Strategies for Late-Stage Parkinson's Disease

Drug treatments and motor complications in advanced-stage Parkinson's disease

Drug strategies for patients with advanced-stage Parkinson's disease

Other symptoms of advanced Parkinson's disease

5 The Role and Indications of Rehabilitation and Physical Therapy in the Treatment of Parkinson's Disease

Re-education in Parkinson's disease: Exercise programs and rehabilitation by disease severity and disability patterns Communication disorders and ENT function

6 Surgical Treatment

Surgical treatments available and inclusion criteria Cost assessment of functional neurosurgery for abnormal movements by stimulation of basal nuclei, particularly the subthalamic nucleus

7 Consensus Document 